\title{
Avaliação dos níveis de compreensão de textos em estudantes universitários
}

\section{Evaluation of levels of texts understanding in university students}

\author{
Adriana Benevides Soares* \\ Docente da Universidade do Estado do Rio de Janeiro - UERJ, Rio de Janeiro, RJ, \\ Brasil
}

\section{Thamires de Abreu Emmerick}

Graduanda em Psicologia pela Universidade do Estado do Rio de Janeiro - UERJ, Rio de Janeiro, RJ, Brasil

\section{Aline Lacerda Vicente}

Graduanda em Psicologia pela Universidade do Estado do Rio de Janeiro - UERJ, Rio de Janeiro, RJ, Brasil

\begin{abstract}
RESUMO
Este estudo objetivou identificar e comparar as diferenças nos níveis de compreensão de textos por universitários. Participaram da pesquisa 312 estudantes de cursos de diferentes áreas do conhecimento, de universidades públicas e privadas. Foram aplicados dois questionários, sendo um de dados sócio-demográficos e outro com questões abertas referentes a um texto. $\mathrm{O}$ questionário foi validado e apresentou Alpha de Crombach de 0,696 . Os principais resultados foram que o maior número de acertos ocorreu nas perguntas referentes ao tema do texto $(62,18 \%)$, seguida de perguntas sobre Detalhes $(56,97 \%)$, seguidas ainda de perguntas sobre as justificativas do texto $(46,58 \%)$ e sobre as inferências $(40,06 \%)$. Constatouse que é no nível de "Macroestrutura" que encontra-se o melhor desempenho dos alunos e que estes são mais eficientes quando pertencem a classes sociais mais altas $(U=5.622 ; p=0.060)$ e freqüentam cursos que exigem maior pontuação para o seu ingresso $(U=13.292 ; p=0.004)$.

Palavras-chave: Compreensão de Textos, Estudantes universitários, Classe social, Curso universitário.
\end{abstract}

\begin{abstract}
This study aimed to identify and compare the difficulties and differences in the understanding level of texts by academics. In the research, 312 students participated, from courses in different areas of knowledge, from public and private universities. Two inquiries were submitted, one of socio-demographic data and the other with free questions concerning a text. The inquiry was certified and presented Alpha Crombach of 0.696. The main findings were that the greatest number of correct answers occurred on questions relating to the subject of the text $(62.18 \%)$, followed by questions about Details $(56.97 \%)$, then by questions about the text's reasoning $(46.58 \%)$ and on the inferences $(40.06 \%)$. It was observed that the best performance of the
\end{abstract}


students were in the "Macrostructure" level and that they are more efficient when belonging to higher social classes $(\bar{x}=70.00 ; p=0.060)$ and attend courses that demand higher scores for admission $(U=392,500 ; p=0,098)$.

Keywords: Text understanding, Students, Social class, University course.

\section{I ntrodução}

"Porque é tão difícil compreender um texto?". Este questionamento tem permeado tanto o campo da Psicologia Cognitiva como também o da Psicologia da Educação, principalmente, no que tange ao meio acadêmico. Segundo Ferreira e Dias (2002) o processo de compreensão de textos requer que o leitor invista no desvelamento e na construção de uma representação para o que ele lê. O processo de compreensão textual envolve importantes processos cognitivos, tais como: percepção, memória, dedução, inferência e a elaboração de estratégias de monitoramento. Desse modo, como apontam Oakhill e Garnham (1988, apud FERREIRA; DIAS, 2002), a leitura proficiente implica na extração do significado, na compreensão, que tem como principal propósito a "aprendizagem da leitura" (p. 51).

Segundo Oliveira e Santos (2005) apesar de a leitura ser uma tarefa comum e corriqueira na contemporaneidade, essa atividade é bem complexa. Leitores pouco habilidosos no exercício da leitura tendem a apenas decodificar os signos do alfabeto, juntar as palavras e as sentenças. Contudo, em uma perspectiva ampla, a leitura requer, necessariamente, que o leitor compreenda o que leu, contextualizando o conteúdo apresentado no texto aos seus conhecimentos de mundo. Apenas decodificar, resulta em uma comprensão de textos insuficiente. De modo complementar, a leitura pode ser vista como um instrumento significativo para a aprendizagem, que permite aos indivíduos conhecer e influenciar o seu entorno (SANTOS; VENDRAMINI; SUEHIRO; SANTOS, 2006).

Tendo em vista a complexidade do processo de compreensão de textos, o objetivo deste estudo foi verificar a compreensão textual de estudantes tendo como base o modelo de compreensão de textos de Kintsch (1998).

\section{Modelo de Construção I ntegração de Kintsch}

Um dos modelos de compreensão mais expressivos na literatura (KINTSCH, 1998; VAN DIJ K; KINTSCH, 1978) busca explicar como as informações do texto e aquelas derivadas do conhecimento anterior do leitor se incorporam e se ligam a uma representação mental coesa. Nesse sentido, o Modelo de Construção Integração $(\mathrm{Cl})$ de Kintsch (1998) merece destaque. Este modelo tem como principal foco o conhecimento de mundo do leitor, os elementos do texto e a coordenação dos componentes de forma e conteúdo. Visando uma melhor organização, Kintsch (1998) preconizou duas fases que 
viabilizam o processo de compreensão: a de construção e a de integração. A fase de construção consiste na construção de um modelo mental mediante o significado das palavras e das proposições do texto. A fase de integração, embora ainda de natureza local, se constitui de forma global e, acontece sempre que uma nova informação é acrescentada. Assim, de forma integrada, o leitor constrói os sentidos do texto a partir de seus conhecimentos prévios e das novas informações obtidas no texto lido. Nesse processo o leitor é ativo, formando uma representação mental coerente com o texto-base e também; através da integração das informações do texto com o seu conhecimento de mundo, forma uma representação mental correspondente às suas significações (SPINILLO; MAHON, 2007; SPINILLO, 2008).

Para Kintsch (1993) um texto pode ser classificado de acordo com três níveis hierárquicos de representação: o nível de superfície, o proposicional e o mais elaborado. No nível de superfície existe uma interação parcial entre autor e leitor. Por conseguinte o leitor tem dificuldades em desvelar o sentido que o autor quis atribuir ao seu texto. O nível proposicional também definido como base do texto constitui o conteúdo semântico coerente do texto. Já o mais elaborado, conhecido também como modelo da situação, incorpora o conteúdo semântico do texto a um conjunto de conhecimentos ou roteiros mentais.

Kintsch (1998) observa que estes três níveis hierárquicos funcionam como um todo, mas para melhor compreender o processo de compreensão textual vale analisá-los de forma mais detalhada.

Segundo van Dijk e Kintsch (1978) a capacidade de compreensão textual envolve tanto a leitura 'visual' quanto a 'auditiva'. Esses dois aspectos podem ser observados tanto em leitores que decodificam facilmente o que lêem quanto em leitores que precisam dedicar mais estratégias e tempo para a decodificação.

No modelo de operações de van Dijk e Kintsch (1978) a estrutura superficial de um discurso é vista como um rol de proposições. Neste rol existe um conjunto de relações semânticas.

O modelo de van Dijk e Kintsch (1983) ainda distingue três níveis de compreensão: o nível de "Macroestrutura", caracterizado como a estrutura geral do texto; o de "Argumentação", que consiste na explicação, na justificativa do autor para o tema do texto; e os "Detalhes", a parte do texto que está como suplemento, acrescentando idéias sem relevância significativa na compreensão do texto. Além desses três níveis, destaca-se também o nível de "Inferência", que requerem conhecimentos extra-texto necessitando de conhecimentos prévios do leitor sobre o tema (VAN DIJK; KINTSCH, 1983).

Segundo van Dijk e Kintsch (1978), o discurso apresenta uma estrutura semântica dividida em dois níveis: microestrutura e 
macroestrutura. A microestrutura é o nível local do discurso, isto é, a estrutura das propostas individuais e suas relações. A macroestrutura tem uma natureza mais global, caracterizando o discurso como um todo. Tanto a microestrutura quanto a macroestrutura constituem um conjunto de regras de mapeamento semântico específico, chamado de macrorregras. Estas reduzem e organizam os detalhes das informações do discurso. Um discurso só é coerente se suas respectivas frases e proposições são interligadas e conectadas, e se essas proposições são organizadas globalmente ao nível de macroestrutura.

Para van Dijk e Kintsch (1978) a estrutura de significado do discurso deve ser identificada como um texto base. Este não é meramente uma lista de proposições não relatadas, mas sim uma unidade coerente e estruturada. Um discurso é identificado como um texto base explícito quando utiliza proposições claras independentes de outras informações do leitor. Não obstante, quando um discurso depende de algumas informações provenientes do conhecimento prévio do leitor, o texto é classificado como um texto base implícito. A estrutura semântica de um discurso deve descrever não apenas os microníveis, mas também os macroníveis. Nesta proposição o texto base deve ser conectado ao que é chamado de topic of discourse, ou seja, o tema geral ou o fragmento dele, estabelecendo assim a idéia global, que concede um significado que caracteriza os termos do discurso. A macroestrutura deve estar atrelada a microestrutura da qual se deriva e para isso é fundamental a análise de algumas macrorregras:

- "deletion" - Cada proposição quando não pode ser direta ou indiretamente interpretada deve ser retirada do texto.

- "generalization" - Cada sequência de proposições podem ser substituídas pela proposição geral sem perder seu significado.

- "construction" - Cada sequência de proposições pode ser substituída pela idéia geral, porém na microestrutura se apresentam as condições, elementos ou consequências das informações.

Para entender o processo de compreensão de textos é importante destacar que um texto possui certos níveis que se relacionam com o nível de compreensão do leitor. Para Rossi (1990) cada parte do texto deve ser considerada relevante, pois o mesmo pode ser visto como um conjunto de objetivos, já que seus elementos informacionais ligam-se aos mesmos. Pode-se dizer então, que um texto pode ser visto como uma composição constituída pela macroestrutura (compreensão do tema geral); argumentação (compreensão dos temas dos parágrafos); e detalhes (compreensão dos elementos de menor importância).

Segundo Kintsch e van Dijk (1978, apud SALLES; PARENTE, 2004) a macroestrutura pode ser compreendida como uma composição de significados que identifica os elementos fundamentais do texto, as 
chamadas macroproposições. $O$ processo de elaboração da macroestrutura do texto tendo como base a microestrutura resultaria então na compreensão.

Ainda no que tange ao nível de macroestrutura, Vilaseca (1999) salienta que o processo de compreensão tem um caráter construtivo, e a macroestrutura consiste na estrutura capaz de apreender as relações semânticas que se estabelecem entre a entrada textual e os conhecimentos prévios que são recuperados na memória no curso do processamento. Como conseqüência desse processo de construção, uma representação proposicional do texto é formulada. Esta inclui as proposições que se formam diretamente a partir da entrada textual, e uma série de conceitos relevantes e irrelevantes.

Vilaseca (1999) ainda pontua que a dificuldade de compreensão dos leitores pouco habilidosos pode estar relacionada com as operações de construção da macroestrutura do texto, ou seja, em como o leitor utiliza seu conhecimento prévio durante o processamento do texto. Portanto, cabe ao leitor esforçar-se no aprimoramento de estratégias cognitivas, que permitirão a integração entre o conhecimento dado e o novo. Deste modo, a macroestrutura será organizada de forma coerente viabilizando o processo de compreensão e aprendizagem.

Ainda que a macroestrutura seja fundamental para a compreensão textual, o processo inferencial é também fundamental. Uma vez que nem tudo está escrito no texto, a formação de inferências é um exercício vital, um processo mental superior dedicado a formação de coerência e organização de significados que permite a recuperação das lacunas nele permeadas (MAHON; SPINILLO, 2007). Para Marcuschi (1996, citado por MAHON; SPINILLO, 2007), as inferências ocupam espaços produzidos pelo autor fazendo com que o texto possa ser compreendido como ainda por terminar sendo resultado de uma múltipla concepção entre o autor e o leitor.

É notório que existem vários processos que implicam na compreensão de textos e não é por acaso que diversos trabalhos são significativos sobre o estudo que concerne deste tema. Nesta vertente, Andrade e Dias (2006) observam a linguagem pela perspectiva da cognição seguindo o arcabouço teórico de Kintsch e van Dijk durante os anos de 1978 a 1983. Estes autores consideram os processos mentais superiores essenciais para a compreensão de textos.

Andrade e Dias (2006) supõem que os indivíduos constituem estruturas consistentes envolvendo aspectos perceptivos e conceituais. Assim, estímulos externos a consciência do leitor são selecionados de acordo com o que é considerado pelo mesmo como coerente ou não. No entanto, a compreensão de textos se torna ainda mais complexa, pois nem sempre o leitor seleciona as informações mais relevantes para uma compreensão global, nem sempre identifica que não está entendendo e nem sempre consegue traçar estratégias para buscar compreender o texto lido. 
Andrade e Dias (2006) concluem que os processos de compreensão de texto requerem que o leitor monitore sua própria leitura e compreensão e produza inferências. Além disso, o leitor precisa integrar a leitura às suas vivências anteriores, dando significado a ela. Diante disso, é fundamental destacar a importância do estudo dos aspectos individuais, sociais e culturais para uma análise eficaz no processo de compreensão de textos.

A partir do arcabouço teórico de Kintsch (1998) foi possível identificar falhas no processo de compreensão textual. E, por sua vez, essas falhas têm dificultado o processo de ensino e aprendizagem pretendido no ensino superior, fato que tem inquietado e desafiado pesquisadores (RODRIGUES; DIAS; ROAZZI, 2002; SILVA; SANTOS, 2004; SANTOS; SUHEIRO; OLIVEIRA, 2004; MARTINS; SANTOS; BARIANI, 2005; OLIVEIRA; SANTOS, 2005; CUNHA; SANTOS, 2006; SANTOS; VENDRAMINI; SUHEIRO; SANTOS, 2006) a se debruçarem sobre essa questão em busca de alternativas de superação para as dificuldades detectadas.

A literatura (MARTINS; SANTOS; BARIANI, 2005; RODRIGUES; DIAS; ROAZZI, 2002) aponta que aspectos como, a classe social e o tipo de curso, podem influenciar a compreensão de textos em universitários. Silva e Santos (2004) constataram que estudantes com maior poder aquisitivo, e que desenvolveram toda a sua vida escolar em escolas particulares, com o apoio de cursinhos pré-vestibular e com grande acesso à leitura, obtiveram um melhor desempenho acadêmico ao longo de sua formação superior. O fato desses estudantes, ter uma melhor condição sócio-econômica, possibilitou que eles tivessem um maior acesso à leitura e às estratégias de compreensão, o que corroborou para um processo de aprendizagem proficiente.

No que concerne à variável tipo de curso, Rodrigues, Dias e Roazzi (2002) verificaram que os alunos de Letras, pela própria característica de constante leitura do curso, tendem a obter resultados mais expressivos no que tange à compreensão textual do que os estudantes de Administração. Além disso, Cunha e Santos (2006) declararam que os alunos de Computação utilizavam menos os recursos de relação entre os nomes do que os alunos de Psicologia, o que evidencia as especificidades e investimentos de cada curso.

Nessa perspectiva, o presente estudo se propôs a identificar as dificuldades e as diferenças nos níveis de compreensão de textos por alunos do Ensino Superior e comparar as dificuldades encontradas por estes alunos. Foi comparado também o desempenho destes alunos em função da classe social e do curso que freqüentavam.

\section{Método}

\section{Participantes}

Participaram desta pesquisa 312 estudantes universitários, sendo 274 alunos da rede pública e 38 da rede privada. Os estudantes 
pertenciam à área de ciências humanas (177 estudantes), ciências exatas (102 estudantes), ciências da saúde ( 25 estudantes), ciências biológicas ( 7 estudantes) e letras e artes ( 1 estudante). A média de idade dos estudantes foi de 22,92 anos, sendo que a idade mínima era de 17 e a máxima de 45 anos. Em relação ao gênero, 105 estudantes eram do gênero masculino e 207 do gênero feminino.

\section{Instrumentos}

Para a coleta de dados da pesquisa foram utilizados dois instrumentos: um questionário para a coleta de dados sóciodemográficos e um questionário sobre um texto narrativo.

O questionário de dados sócio-demográficos registrou informações sobre os participantes: gênero, idade, tipo de universidade (pública ou privada), semestre letivo e curso realizado pelo estudante.

0 instrumento 2 , texto e seu respectivo questionário com um total de 17 questões discursivas: 5 questões sobre "Macroestrutura", 4 questões sobre "Argumentação", 4 questões sobre "Detalhes" e 4 questões sobre "Inferências".

Foi feita uma correlação bisserial item a item para a validação do questionário a ser aplicado nesta pesquisa. O coeficiente de correlação bisserial também foi utilizado devido ao tipo de instrumento proposto, ou seja, um questionário havendo uma dicotomização. Esse instrumento contemplava 20 perguntas, onde o escore foi dividido em certo e errado. A informação sobre o item no sentido de prognosticar bem ou não em relação a um escore total foi analisado tendo como base um ponto de corte 0,25 . As questões $2,3,9,16,17$ e 20 não revelam significância, mas ao mesmo tempo, não foram descartadas, pois a diferença é pequena em relação a correlação bisserial recomendada $(<\mathrm{ou}=\mathrm{a} 0,05)$. No entanto, três itens foram retirados por estarem fora do padrão, perfazendo um total de 17 itens. Esses itens descartados referem-se a uma pergunta de detalhe, uma de inferência e outra de argumentação. A retirada dos itens não prejudicou a análise dos dados.

Em uma nova aplicação do instrumento 2, nos 312 universitários, foi possível determinar a validade do construto, a dimensionalidade e a consistência interna. Foram efetuadas análises de itens, análise fatorial, correlação bisserial e a avaliação da consistência interna do instrumento. O cálculo do poder de discriminação dos itens, a partir da correlação entre cada item, e o escore total revelou que todos os itens estavam adequados. A consistência interna foi considerada satisfatória uma vez que o Alpha de Crombach foi de 0.696.

\section{Procedimentos}

Os dois instrumentos foram aplicados a cada estudante universitário, sendo sempre precedidos por um termo de consentimento livre e esclarecido sobre a pesquisa. 
A aplicação dos instrumentos foi, em sua maioria, coletiva, com a autorização dos docentes e diretores dos cursos universitários, e, em algumas poucas vezes, de forma individual, em apenas uma sessão e sem tempo determinado. Os participantes foram previamente alertados sobre a finalidade da pesquisa e avisados de que poderiam consultar o texto quantas vezes julgassem necessário. É importante ressaltar que só participaram da pesquisa os estudantes que assinaram o Termo de Consentimento Livre e Esclarecido.

\section{Resultados}

Mediante a análise estatística dos dados obtidos com a pesquisa foi possível verificar, como mostra a Tabela 1, que o maior número de acertos (em termos de média percentual) ocorrem nas perguntas referentes a "Macroestrutura" (62,18\%), seguida de "Detalhes" (56,97\%), "Argumentação" (46,58\%) e "Inferência" (40,06\%).

Tabeh 1: Estatisticas referentes ao percentual de Acerto nos núveis

\begin{tabular}{ccccc}
\hline & Argumentação & Detalhe & Macroestrutura & Inferência \\
\hline Média & 46.58 & 56.97 & 62.18 & 40.06 \\
Mediana & 33.33 & 50.00 & 60.00 & 40.00 \\
Desvio Padrão & 33.21 & 22.09 & 28.61 & 18.18 \\
\hline
\end{tabular}

A partir da análise do teste de Análise de Variância de Friedman ou teste de Friedman, também conhecida como Dupla Análise de Variância por Postos no intuito de comparar o desempenho nos níveis (macroestrutura, argumentação, inferências, e detalhes) foi possível verificar, como mostra a Tabela 2, que não há diferenças significativas entre os níveis Argumentação e Inferência; Detalhes e Macroestrutura. 
Tabela 2: Análise de Variância de Friedman

\begin{tabular}{lcccc}
\hline & Argumentação & Detalhe & Macroestrutura & Inferência \\
\hline Soma dos Ranks & 691.00 & 871.50 & 947.50 & 610.00 \\
Média dos Ranks & 2.21 & 2.79 & 3.04 & 1.95 \\
X2 de Friedman & 140.86 & & & \\
Graus de liberdade & 3 & & & \\
p & 0.000 & & \\
\hline Comparaçőes: & Diferença & $\mathbf{p}$ & \\
\hline Argu e Detal & 180.50 & $<0.05$ & \\
Argu e Macro & 256.50 & $<0.05$ & \\
Argu e Infer & 81.00 & Não significativo & \\
Detal e Macro & 76.00 & Não significativo & \\
Detal e Infer & 261.50 & $<0.05$ & \\
Macro e Infer & 337.50 & $<0.05$ & \\
\hline
\end{tabular}

Para identificar nesses níveis, quais as classes sociais cujas diferenças foram significativas, utilizou-se o teste U de Mann-Whitney. A Tabela 3 apresenta a relação entre as Classes Sociais e os Níveis.

Tabela 3: Classes Sociais e Níveis

\begin{tabular}{|c|c|c|c|c|c|c|}
\hline Nível & CLASSE SOCIAL & $\mathbf{N}$ & $\begin{array}{c}\text { Média } \\
(\%)\end{array}$ & $\begin{array}{l}\text { Posto } \\
\text { Médio }\end{array}$ & $\mathbf{U}$ & $\mathbf{p}$ \\
\hline \multirow[t]{3}{*}{ Axgum entação } & Baix a & 60 & 43.89 & 148.66 & 0.539 & 0.764 \\
\hline & Média & 237 & 47.26 & 157.69 & & \\
\hline & Alta & 14 & 47.62 & 158.79 & & \\
\hline \multirow[t]{3}{*}{ Detalhes } & Baix a & 60 & 49.58 & 130.59 & 6.950 & 0.031 \\
\hline & Média & 237 & 58.65 & 162.35 & & \\
\hline & Alta & 14 & 58.93 & 157.46 & & \\
\hline \multirow[t]{3}{*}{ Macroestrutura } & Baix a & 60 & 54.67 & 132.80 & 5.622 & 0.060 \\
\hline & Média & 237 & 63.46 & 160.62 & & \\
\hline & Alta & 14 & 70.00 & 177.14 & & \\
\hline \multirow[t]{3}{*}{ Inferência } & Baix a & 60 & 33.67 & 127.83 & 18.166 & 0.000 \\
\hline & Média & 237 & 40.84 & 158.65 & & \\
\hline & Alta & 14 & 54.29 & 231.86 & & \\
\hline \multirow[t]{3}{*}{ Total } & Baix a & 60 & 45.39 & 127.23 & 8.876 & 0.012 \\
\hline & Média & 237 & 52.82 & 161.38 & & \\
\hline & Alta & 14 & 58.82 & 188.14 & & \\
\hline
\end{tabular}


No nível Detalhes foi significativa a comparação entre a classe Baixa e Média, com a classe média obtendo maior percentual de acertos ( $U$ $=5660.00 ; \mathrm{p}=0.008$ ).

No nível Inferência foram significativas todas as comparações, com a classe Baixa obtendo menor percentual de acertos do que a classe Média e Alta, e a classe Média obtendo menor percentual de acertos do que a classe Alta.

No total foram significativas as comparações entre a classe Baixa e Média e entre a classe Baixa e Alta, sempre com a classe Baixa obtendo menor percentual de acertos do que as demais.

Para identificar nesses níveis, quais as áreas cujas diferenças foram significativas utilizou-se o teste $U$ de Mann-Whitney.

Tabela 4: Nír eis de compreensão do texto e áreas dos cursos

\begin{tabular}{|c|c|c|c|c|c|c|}
\hline Nivel & AREA & $\mathbf{N}$ & $\begin{array}{c}\text { Média } \\
(\%)\end{array}$ & $\begin{array}{l}\text { Posto } \\
\text { Médio }\end{array}$ & $\mathbf{U}$ & $\mathbf{p}$ \\
\hline \multirow[t]{4}{*}{ Argumentação } & Humanas & 176 & 44.51 & 150.88 & 5.896 & 0.117 \\
\hline & Saúde & 27 & 49.38 & 164.00 & & \\
\hline & Exatas & 102 & 50.98 & 168.18 & & \\
\hline & Biológicas & 7 & 23.81 & 98.79 & & \\
\hline \multirow[t]{4}{*}{ Detalhes } & Humanas & 176 & 55.40 & 152.66 & 1.795 & 0.616 \\
\hline & Saúde & 27 & 56.48 & 149.46 & & \\
\hline & Exatas & 102 & 60.05 & 165.49 & & \\
\hline & Biológicas & 7 & 53.57 & 149.14 & & \\
\hline \multirow[t]{4}{*}{ Macroestrutura } & Humanas & 176 & 58.75 & 145.92 & 13.292 & 0.004 \\
\hline & Saúde & 27 & 74.07 & 191.61 & & \\
\hline & Exatas & 102 & 66.47 & 170.09 & & \\
\hline & Biológicas & 7 & 40.00 & 88.93 & & \\
\hline \multirow[t]{4}{*}{ Inferência } & Humanas & 176 & 40.11 & 156.72 & 6.026 & 0.110 \\
\hline & Saúde & 27 & 46.67 & 187.41 & & \\
\hline & Exatas & 102 & 38.82 & 151.07 & & \\
\hline & Biológicas & 7 & 31.43 & 111.00 & & \\
\hline \multirow[t]{4}{*}{ Total } & Humanas & 176 & 49.97 & 147.78 & 9.833 & 0.020 \\
\hline & Saúde & 27 & 57.52 & 183.76 & & \\
\hline & Exatas & 102 & 54.09 & 168.84 & & \\
\hline & Biológicas & 7 & 37.82 & 90.86 & & \\
\hline
\end{tabular}

De acordo com a Tabela 4, pode-se perceber que no nível Macroestrutura foi significativa a comparação entre Humanas e Saúde, e Humanas e Exatas, com a área de Humanas obtendo menor percentual de acertos. Foi significativa, também, a comparação entre 
Biológicas e Saúde, e Biológicas e Exatas, com a área de Biológicas obtendo menor percentual de acertos.

No total foram significativas as comparações entre Biológicas e Saúde, e Biológicas e Exatas, com a área de Biológicas obtendo menor percentual de acertos.

\section{Discussão e Considerações Finais}

O processo de compreensão de textos na universidade é uma habilidade fundamental a ser desenvolvida, pois é por intermédio da leitura que o estudante universitário pode aprender o conteúdo ministrado nas diversas disciplinas concernentes ao seu curso e também construir suas hipóteses de estudo (SANTOS; SUHEIRO; OLIVEIRA, 2004). Somente mediante uma leitura e compreensão proficientes que os estudantes podem ter um desempenho acadêmico satisfatório.

Os principais resultados obtidos nesta pesquisa mostraram que o maior número de acertos ocorreu nas perguntas referentes à "Macroestrutura" (62,18\%), seguida de "Detalhes" (56,97\%), "Argumentação" (46,58\%) e "Inferência" (40,06\%). Tais dados permitem afirmar que o nível de compreensão em leitura dos universitários mostrou-se aquém do desejado e esperado nos níveis de compreensão de texto referentes à "Argumentação" $(46,58 \%)$ e à "Inferência" $(40,06 \%)$. Este fato pode ser entendido a medida em que compreender um texto implica em adentrar em uma relação causal organizando os acontecimentos e ações estruturantes e ligando as informações anteriores do leitor às novas oferecidas pelo texto (MIGUEL, 2002). Neste âmbito, muitos leitores têm dificuldade em entender o que o texto argumenta e em estabelecer inferências, relações entre os conhecimentos pré-estabelecidos e os novos.

Apesar das dificuldades encontradas nos níveis de "Argumentação" e "Inferência", pode-se observar que é no nível de base do texto ("Macroestrutura") que encontra-se o melhor desempenho dos alunos e que este desempenho é mais eficiente quando estes pertencem a classes sociais mais altas e freqüentam os cursos que exigem maior pontuação para o seu ingresso.

Como foi possível observar na Tabela 2, não foram encontradas diferenças significativas entre os níveis Argumentação e Inferência; Detalhes e Macroestrutura. Contudo, no que tange à comparação entre os outros níveis (Argumentação e Detalhes; Argumentação e Macroestrutura; Detalhes e Inferência; e, Macroestrutura e Inferência) constatou-se diferenças significativas ( $p<0.05)$.

Esses dados evidenciam que, na verdade, muitos estudantes chegam à universidade sem compreender um texto em sua totalidade. Kintsch (1998) alerta para o fato de que selecionar as informações do texto não implica em entendimento ou compreensão do mesmo. Ou seja, o 
fato de selecionar algumas sentenças do texto não leva à compreensão do texto como um todo.

Nesse sentido, muitos universitários podem ser considerados como compreendedores ineficientes, pois como observamos com os dados observados neste trabalho, embora sejam capazes de responder de forma correta às questões sobre a macroestrutura textual não conseguem, muita das vezes, compreender sua argumentação.

Quando um leitor não consegue compreender a argumentação de um texto, conseqüentemente, também tem dificuldades em estabelecer inferências sobre o texto lido. Miguel (2002) destaca essa carência de relação entre o autor e o leitor como um problema de compreensão. É a interação entre o que o autor argumenta e inferências do leitor, que permite àquele que lê atingir uma compreensão rasteira ou elaborada do texto. Na compreensão rasteira é possível sintetizar ou rememorar o conteúdo, mas é na compreensão mais elaborada que é possível resolver problemas inéditos, já que há uma conexão do conhecimento anterior do leitor com as informações fornecidas pelo texto.

Todas as comparações realizadas destacaram ainda que a classe Baixa obteve menor percentual de acertos do que as classes Média e Alta. De acordo com Silva e Santos (2004) essa evidência pode ser explicada pelo fato de que os estudantes de classe social mais alta advém, na maior parte das vezes, de escolas particulares e que realizaram escolas preparatórias para serem aprovados em cursos bastante disputados são compreendedores de texto mais proficientes do que aqueles de classe social mais baixa.

Silva e Santos (2004) destacam ainda que alunos melhor preparados e bem treinados em suas capacidades e na utilização de estratégias para o acesso a universidade tendem a obter um melhor desempenho acadêmico, o que sustenta a hipótese de que o acesso à leitura e a freqüência da leitura ao longo do desenvolvimento do aluno podem ter influído nestes resultados.

Quanto à área do curso, as diferenças significativas foram encontradas no nível de "Macroestrutura", sendo que os estudantes de Ciências Humanas apresentaram desempenho inferior às áreas de Exatas e Biomédicas. Este dado é, de certa forma, surpreendente, pois segundo algumas pesquisas anteriores (CUNHA; SANTOS, 2006; MARTINS; SANTOS; BARIANI, 2005), esperava-se que os universitários da área de Humanas tivessem melhor desempenho na compreensão leitura, já que pressupõe-se que estes, pela própria característica do curso, teriam maior interesse pela leitura e, conseqüentemente, uma maior facilidade na compreensão.

Uma hipótese para este resultado pode ser atribuída ao fato, como outrora sustentado por Silva e Santos (2004), de que os alunos que tiveram uma alta nota de corte para o ingresso no curso Superior tenderiam a compreender textos com maior proficiência. Estes alunos 
teriam desenvolvido habilidades de raciocínio e inferência que lhes teria permitido ter melhor proficiência na leitura. Esta hipótese ganha relevância no presente estudo na medida em que este contou com uma amostra bastante expressiva de estudantes de instituições públicas, cujas notas de corte para os cursos referentes à área de Exatas e Biológicas costumam ser elevadas.

Diante dos resultados obtidos é possível refletir que é cada vez maior o número de indivíduos que são alfabetizados, mas que não conseguem compreender o código que lêem. Entre esses sujeitos estão inclusive estudantes universitários que acessam aos cursos de nível superior sem o desempenho em compreensão de textos esperado (MARTINS; SANTOS; BARIANI 2005). Ao cursar a formação superior espera-se que o aluno seja capaz de ler perseguindo objetivos, de criar suas estratégias pessoais de compreensão, de resumir a informação a partir do texto e de seu próprio próprio conhecimento de mundo, de adquirir uma postura reflexiva face à informação, de realizar inferências e, enfim, de aplicar o conhecimento em problemas práticos (VICENTILLI, 1999 apud MARTINS; SANTOS; BARIANI 2005). No entanto, pesquisas (OLIVEIRA; SANTOS, 2005; CUNHA; SANTOS, 2006; RODRIGUES; DIAS; ROAZZI, 2002) têm demonstrado o baixo nível de habilidade na compreensão textual em estudantes universitários.

O que fazer diante deste quadro? Silva e Santos (2004) sugerem que as universidades e seus professores se preparem para colaborar com a aprendizagem de seus alunos, reconhecendo, primeiramente, suas fragilidades e potencialidades para que estratégias de compreensão de textos eficazes sejam implementadas. Para isso é importante que diagnósticos pontuais sobre a qualidade do ensino Superior sejam delineados para que estratégias educacionais adequadas sejam realizadas de acordo com a formação específica e visando formar profissionais autônomos e independentes.

\section{Referências Bibliográficas}

ANDRADE, M. W. C. L.; DIAS, M. G. B. Processos que levam à compreensão de textos. Psicologia em Estudo, v. 11, n. 1, p. 147154, 2006.

CUNHA, N. B.; SANTOS, A. A. A. Relação entre a compreensão da leitura e a produção escrita em universitários. Psicologia: Reflexão e Crítica, v. 19, n. 2, p. 237-245, 2006.

FERREIRA, S. P. A.; DIAS, M. G. B. B. Compreensão de leitura: estratégias de tomar notas e da imagem mental. Psicologia: Teoria e Pesquisa, v. 18, n. 1, p. 51-62, 2002.

KINTSCH, W. Information accretion and reduction in text processing: Inferences. Discourse Processes, v. 16, p. 193-202, 1993.

Comprehension: A paradigm for cognition. Cambridge: University Press, 1998. 
MARTINS, R. M. M.; SANTOS, A. A. A.; BARIANI, I. C. D. Estilos cognitivos e compreensão leitora em universitários. Paidéia, v. 11, n. 1, p. 147-154, 2006.

MIGUEL, E. S. Compreensão e redação de textos: dificuldades e ajudas. Porto Alegre: Artmed, 2002.

OLIVEIRA, K. L.; SANTOS, A. A. A. Compreensão em leitura e avaliação da aprendizagem em universitários. Psicologia: Reflexão e Crítica, v. 8, n. 1, p. 118-124, 2005.

RODRIGUES, A. A.; DIAS, M. G. B. B.; ROAZZI, A. Raciocínio Lógico na compreensão de texto. Estudos de Psicologia (Natal), v. 7, n. 1, p. 117-132, 2002.

ROSSI, J. P. The function of frame in the comprehension of scientific text. J ournal of Education Psychology, v. 82, p. 727-732, 1990. SALLES, J. F.; PARENTE, M. A. P. P. Compreensão textual em alunos de segunda e terceira séries: uma abordagem cognitiva. Estudos de Psicologia (Natal), v. 9, n. 1, p. 71-80, 2004.

SANTOS, A. A. A.; SUEHIRO, A. C. B.; OLIVEIRA, K. L. Habilidades em compreensão da leitura: um estudo com alunos de psicologia. Estudos de Psicologia (Campinas), v. 21, n. 2, p. 41-49, 2004. SANTOS, A. A. A.; VENDRAMINI, C. M. M.; SUEHIRO, A. C. B.; SANTOS, L. A. D. Leitura compreensiva e utilização de estratégias de aprendizagem em alunos de Psicologia. Estudos de Psicologia, Campinas, v. 23, n. 1, p. 83-91, 2006.

SILVA, M. J. M.; SANTOS, A. A. A. A avaliação da compreensão em leitura e o desempenho acadêmico de universitários. Psicologia em Estudo, v. 9, n. 3, p. 459-467, 2004.

SPINILLO, A. G. O leitor e o texto: desenvolvendo a compreensão de textos na sala de aula. Revista Interamericana de Psicologia, v. 42 , n. 1, p. 29-49, 2008.

SPINILLO, A. G.; MAHON, E. R. Compreensão de textos em crianças: comparações entre diferentes classes de inferência a partir de uma metodologia on-line. Psicologia: Reflexão e Crítica, v. 20, n. 3, p. 463-471, 2007.

VAN DIJK, T. A.; KINTSCH, W. Toward a model of text comprehension and production. Psychology Review, v. 85, n. 5, p. 363-394, 1978.

VILASECA, J. L. L. La construcción de la macroestructura: de comprensión al aprendizaje a partir de textos. Comprensión lectora y memoria operativa. Aspectos evolutivos e instruccionales. Barcelona: Paidós, 1999, p. 1-56.

\section{Endereço para correspondência}

Adriana Benevides Soares

Universidade de Estado do Rio de Janeiro, UERJ, Rua São Francisco Xavier, 524, 10ㅇaㅁ, Bloco B, sala 10.019, Maracanã, CEP 2550-013, Rio de Janeiro - RJ, Brasil

Endereço eletrônico: adribenevides@gmail.com 
Thamires de Abreu Emmerick

Universidade de Estado do Rio de Janeiro, UERJ, Rua São Francisco Xavier, 524, 10 andar, Bloco B, Secretaria da Graduação, Maracanã, CEP 2550-013, Rio de Janeiro - RJ, Brasil

Endereço eletrônico: thataemmerick@hotmail.com

Aline Lacerda Vicente

Universidade de Estado do Rio de Janeiro, UERJ, Rua São Francisco Xavier, 524, 10 andar, Bloco B, Secretaria da Grauduação, Maracanã, CEP 2550-013, Rio de J aneiro - RJ, Brasil

Endereço eletrônico: lynka22@yahoo.com.br

Recebido em: 19/10/2009

Aceito para publicação em: 15/04/2010

Acompanhamento do processo editorial: Rita Maria Manso de Barros

\section{Notas}

* Psicóloga; Doutora em Psicologia Cognitiva pela Universidade de Paris XI; Docente da graduação e da pós-graduação da Universidade Salgado de Oliveira - UNIVERSO e da Universidade do Estado do Rio de Janeiro - UERJ. 